\title{
Giant Malignant Cystic Pheochromocytoma: A Case Report
}

\author{
Mehdi Soufi • Mohammed K. Lahlou • Said Benamr • \\ Rahal Massrouri • Jalil Mdaghri • Abdelhamid Essadel • \\ Mohammadine El Hamid • Ahmed Taghy • \\ Abdelatif Settaf • Bouziane Chad
}

Received: 30 June 2009 / Accepted: 6 October 2009/Published online: 12 August 2012

(C) Association of Surgeons of India 2012

\begin{abstract}
Cystic malignant pheochromocytomas are uncommon. Differing from solid pheochromocytomas, which produce catecholamines and present adrenergic syndrome, cystic pheochromocytomas, may not produce these. Preoperative diagnosis may be difficult. Ct scan is useful for preoperative management. In this report, we describe a case of a giant malignant cystic pheochromocytoma in a young woman (17 years old) which presented as giant abdominal mass. The malignancy was confirmed by the presence of liver metastasis. Two years after curative resection, the patient is in good health with no recurrence.
\end{abstract}

Keywords Neuroendocrine tumors $\cdot$ Malignant

Pheochromocytoma $\cdot$ Adrenalectomy $\cdot$ Chemotherapy

\section{Introduction}

Malignant pheochromocytomas affecting young people are rare. They rarely presented as predominantly cystic masses [1]. The histopathological evaluation cannot distinguish between benign and malignant tumours. Few cases of cystic pheochromocytomas have been reported in the literature review. In this case, we describe a malignant cystic pheochromocytoma which presented as an abdominal mass in a young 17 years old woman.

\section{Soufi $(\bowtie)$}

Faculty of Medicine, University Mohammed First Oujda Morocco, BP 4847 Oujda University,

Oujda 60049, Morocco

e-mail: drsoufimehdi@hotmail.fr

M. K. Lahlou · S. Benamr $\cdot$ R. Massrouri $\cdot$ J. Mdaghri •

A. Essadel • M. El Hamid · A. Taghy · A. Settaf • B. Chad

Surgery B, Avicenne lbn sina Hospital,

Rabat, Morocco

\section{Case Report}

A 17 years old woman was referred for evaluation of an abdominal mass. Physical examination revealed a slightly tender mass of $20 \mathrm{~cm}$ mass in the upper right quadrant. Basic Cardio vascular examination was normal. Ultrasonography examination showed a giant heterogenic and cystic mass. A computed tomography (CT) scan revealed a big mass in the left adrenal gland with dimensions of $21 \times 15 \mathrm{~cm}$ (Fig. 1). This mass seemed invading the right kidney. There was central necrosis and peripheral calcifications (Fig. 2). The laboratory tests were all within normal limits (carcinoembryonic antigen, CA19-9, CA125, hydatid serology, alpha-fetoprotein levels, and 24-hour assays for catecholamines and their metabolites). At surgical exploration, a $20 \mathrm{~cm}$ fixed round well-vascularized retroperitoneal mass that involved the right kidney and $1 \mathrm{~cm}$ of the liver nodule was found. The patient suddenly suffered a myocardial infarction suppressed under medical treatment. En bloc right radical nephrectomy and lymph node dissection was performed. Histological analysis showed a solid-cystic adrenal neuroendocrine tumor that involved the right kidney. There was no lymphatic metastasis. The result from immunohistochemical staining confirmed the presence, in specimen resection and in liver nodule, of malignant pheochromocytoma. There were no post-operative complications. MIBG was not done due to its non availability. A combination of chemotherapeutic regimen was prescribed, consisting of Doxorubicin, Cyclophosphamide, and Vincristine. It was stopped after three courses because of its toxicity. Two years after the surgery, our patient is still alive, with no recurrence or distant metastasis.

\section{Discussion}

First described in 1886 by Fränkel, pheochromocytomas are rare catecholamine-secreting tumors derived from the 


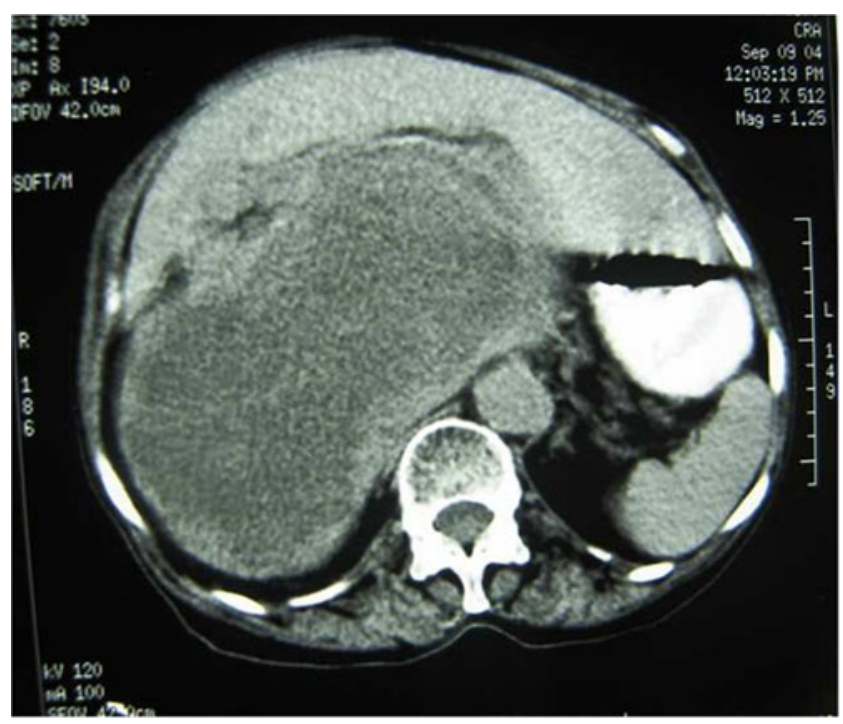

Fig. 1 CT scan showing a giant pheochromocytoma pushing the veina cava and the rignt hepatic lobe

chromaffin cells of the embryonic neural crest [2]. Malignant pheochromocytomas are rare. As with other neuroendocrine tumors, the diagnosis of malignancy is not primarily based on cytological characteristics, but is defined by the presence of local invasion or metastatic disease. The presence of metastases of chromaffin tissue at sites where no chromaffin tissue should be expected provides the only currently widely accepted means of defining malignancy

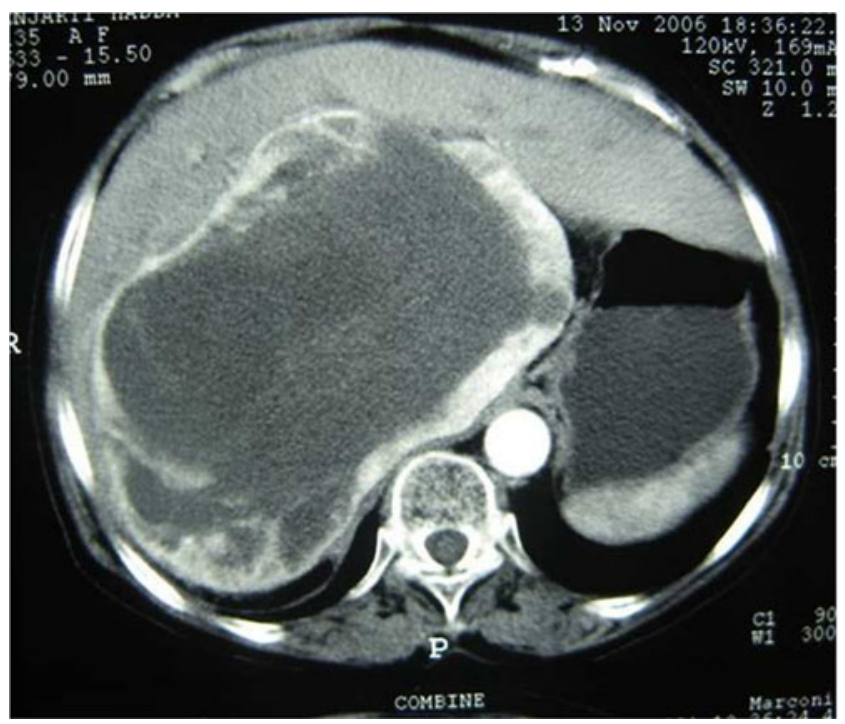

Fig. 2 CT Scan with intraarterial injection of contrast revealing a huge rignt pheochromocytoma, with peripheral contrast enhancement and cystic, necrotic and calcifications components
[3]. In our case, the discovery of small liver metastasis confirms the diagnosis of malignancy [3]. The incidence of metastatic pheochromocytoma is between $13 \%$ and $26 \%$ [4]. Pheochromocytomas occur most frequently among adults. Children constitute $10 \%$ of the affected people. Cystic giant and no secreting pheochromocytomas are rarely reported in these cases [5]. Our patient presents an abdominal mass without any clinical sign. In pheochromocytoma, the classic triad presentation is episodic headache, sweating, and palpitations [6]. Once a pheochromocytoma is suspected, the diagnosis is based on demonstration of catecholamine overproduction by measurement of urine or plasma catecholamines and their metabolites. These metabolites were negative in our patient, from where the difficulty of diagnosis. In these cases, the radiological findings are helpful for the diagnosis. Ultrasound should usually be employed as a first exam. In our case, it evoked a hydatid cyst of liver because of the size and the cystic component of lesion [7]. CT scan and MRI are sensitive to localize the tumor. Abdominal CT has high sensitivity to suggest malignancy [7]. Because there is currently no effective cure for malignant pheochromocytoma, most treatments are palliative; however, in some cases, tumour and metastasis resection, if resectable, prolong survival and reduce exposure of the cardiovascular system [4]. Alternatives to surgical resection include external beam radiation, cryoablation, radiofrequency ablation, and chemotherapy [8]. Treating metastatic lesions with therapeutic doses of iodine-131 MIBG give good results [8]. Without treatment, the 5-year survival is generally around $50 \%$ [4].

\section{Conclusion}

The giant asymptomatic and non secreting pheochromocytoma is rare. The big size of the tumour, the excessive excretion of dopamine, and the local extension are arguments in favour of the malignancy. We would like to emphasize the importance of preoperative diagnosis and performing radiographics that can establish the right diagnosis in non secreting giant pheochromocytoma. By doing that, we can reduce the mortality and the complications related to the disease. The treatment in these forms requires a multidisciplinary management.

Competing Interests The authors declare that they have no competing interests. 


\section{References}

1. Waqar AN, Sarwar A (2008) Metastatic malignant pheochromocytoma of adrenal gland. J Coll Phys Surg Pakistan 18(5):305-307

2. Frankel F (1886) Classics in oncology. A case of bilateral completely latent adrenal tumor and concurrent nephritis with changes in the circulatory system and retinitis. CA Cancer J Clin 1984(34):93-106

3. Glodny B, Winde G, Herwig R et al (2001) Clinical differences between benign and malignant pheochromocytomas. Endocr $\mathrm{J}$ 48:151-9
4. Eisenhofer G, Bornstein SR, Brouwers FM et al (2004) malignant pheochromocytoma: current status and initiative for future progress. Endocr Relat Cancer 11:423-36

5. Ciftci AO, Cahit FT et al (2002) Pheochromocytoma in children. J Pediatr Surg 36(3):447-452

6. Manger WM (2005) The vagaries of pheochromocytomas. Am J Hypertens 18:1266-1270

7. Lee TH, Slywotzky CM, Lavelle MT et al (2002) Cystic pheochromocytoma. Radiographics 22(4):935-40

8. Sisson JC, Shapiro B, Shulkin BL et al (1999) Treatment of malignant pheochromocytomas with 131I metaiodobenzylguanidine and chemotherapy. Am J Clin Oncol 22:364-70 